\title{
Naringenin inhibits transforming growth factor- $\beta 1$-induced cardiac fibroblast proliferation and collagen synthesis via G0/G1 arrest
}

\author{
MINGXIN LIU ${ }^{1}$, XIPING XU ${ }^{1}$, JIANHUA ZHAO ${ }^{1}$ and YANHONG TANG ${ }^{2-4}$ \\ ${ }^{1}$ Department of Cardiology, The First People's Hospital of Yueyang, Yueyang, Hunan 414000; \\ ${ }^{2}$ Department of Cardiology, Renmin Hospital of Wuhan University; ${ }^{3}$ Cardiovascular Research Institute, \\ Wuhan University; ${ }^{4}$ Hubei Key Laboratory of Cardiology, Wuhan, Hubei 430060, P.R. China
}

Received July 15, 2016; Accepted June 29, 2017

DOI: $10.3892 / \mathrm{etm} .2017 .5103$

\begin{abstract}
The Traditional Chinese Medicine naringenin (NRG) has a number of biological effects, including anti-inflammatory, anti-oxidative, anti-tumor and anti-atherosclerotic effects. However, the mechanism underlying its effects remains unclear. The aim of the present study is to investigate the role and mechanism of NRG on proliferation and collagen synthesis of cardiac fibroblasts (CFs) induced by transforming growth factor $\beta 1$ (TGF- $\beta 1$ ). Firstly, proliferation and collagen synthesis in CFs subjected to TGF- $\beta 1$ was assessed subsequent to the consumption of NRG or control treatment. Additionally, the cell cycle of different groups and the roles of cyclins and cyclin-dependent kinases (CDKs) in NRG treatment of CFs were detected. In the present study, it was revealed that treatment of CFs with NRG resulted in attenuated fibroblast $\alpha$-smooth muscle actin expression, deceased proliferation and collagen synthesis when compared with a TGF- $\beta 1$ stimulus. Additionally, it was demonstrated that cell population of CFs treated with NRG in the S-phase became smaller whereas that of CFs in the G0/G1-phase increased when compared with the TGF- $\beta 1$ group. Mechanistically, the expression of cyclin D1-CDK4/6 and cyclin E2-CDK2 were inhibited in the NRG treatment group. These results illustrated that the protective effects of NRG on proliferation and collagen synthesis of CFs were at least in part due to G0/G1 arrest. Therefore, NRG may become a novel strategy for treating cardiac fibrosis in the future.
\end{abstract}

Correspondence to: Dr Yanhong Tang, Department of Cardiology, Renmin Hospital of Wuhan University, 238 Jiefang Road, Wuhan, Hubei 430060, P.R. China

E-mail: wurmheart@vip.163.com

Key words: naringenin, cardiac fibroblasts, proliferation, collagen synthesis, cell cycle

\section{Introduction}

Cardiac fibrosis refers to excessive accumulation of collagen in the normal myocardium and significantly increased collagen concentration or change of collagen composition in heart tissue. Cardiac fibrosis is the inevitable process of a variety of clinical cardiovascular diseases that have developed to the final stage, which is the main performance of cardiac remodeling $(1,2)$. At present, it is known that cardiac fibrosis is closely associated with arrhythmia, heart failure and mortality due to sudden cardiac arrest (3-5). It is generally known that activation and proliferation of cardiac fibroblasts $(\mathrm{CFs})$ and deposition of extracellular matrix (ECM) secreted by CFs are the primary features of cardiac fibrosis $(1,6)$. Accumulation of ECM increases myocardial hardness, reduces myocardial compliance and affects the normal diastolic and systolic function of the heart (6). Therefore, cardiac fibrosis is a key factor in cardiovascular disease outcomes, and CFs are important in the process of cardiac fibrosis (6). In the stimuli of myocardial infarction, an excessive pressure load or neurohumoral factors and $\mathrm{CFs}$ begin with pathologic hyperplasia and convert to myofibroblasts $(6,7)$. Under the mediation of a variety of cytokines, myofibroblasts can migrate to the damaged area and proliferate rapidly to synthesize and release a large amount of collagen I and III, which accumulates in the myocardial interstitium and perivascular spaces (3). It may also promote crosslinking between the ECM, and cause excessive deposition of collagen that leads to cardiac fibrosis and provides a pathological basis to the occurrence and development of cardiovascular diseases $(6,8)$. Therefore, in improving the prognosis of cardiovascular diseases and inhibiting cardiac remodeling at the cellular level, $\mathrm{CFs}$ have become an important target to control the progression of cardiac fibrosis. Abrogation of CF transformation into myofibroblasts, and the inhibition of CF proliferation and collagen synthesis may be a strategy for suppressing cardiac fibrotic remodeling.

Naringenin (NRG) is a type of double hydrogen flavonoid that predominantly exists in rutaceae citrus plants (9). A number of studies have confirmed that NRG demonstrates numerous biological effects on oxidative stress, the inflammatory response, disorders of lipid metabolism and apoptosis (9-11). 
Recently, the cardioprotective effects of NRG have been the focus of a number of studies $(12,13)$. However, the mechanism underlying its effects remains unclear. Therefore, the aim of the present study was to investigate the effects and mechanism of NRG on the proliferation and collagen synthesis of CFs induced by transforming growth factor $\beta 1$ (TGF- $\beta 1$ ) from the cellular level to elucidate the mechanism of NRG against cardiac fibrosis.

\section{Materials and methods}

Materials. Primary antibodies including anti-cyclin D1 (cat no. 2978), anti-cyclin dependent kinase 4 (anti-CDK4; cat no. 12790), anti-CDK6 (cat no. 3136) and anti-CDK2 (cat no. 2546) were purchased from Cell Signaling Technology, Inc. (Danvers, MA, USA). Primary anti-cyclin E2 antibody (cat. no. sc-22777) was purchased from Santa Cruz Biotechnology, Inc. (Dallas, TX, USA). Primary anti-GAPDH antibody (cat. no. MB001) was purchased from Bioworld Technology, Inc. (St. Louis Park, MN, USA). Cell counting kit-8 (CCK-8; cat. no. CK04-11) was purchased from Dojindo Molecular Technologies, Inc. (Rockville, MD, USA). TRIzol reagent (cat. no. 15596018) was purchased from Invitrogen (Thermo Fisher Scientific, Inc., Waltham, MA, USA). Transcriptor First Strand cDNA Synthesis kit (cat no. 04896866001) was bought from Roche Diagnostics (Basel, Switzerland). Bicinchoninic acid (BCA) protein assay kit (cat. no. 23227) was from Pierce (Thermo Fisher Scientific, Inc.). NRG (cat. no. W530098) was purchased from Sigma-Aldrich (Merck KGaA, Darmstadt, Germany).

Cell culture and treatment. A total of 70 neonatal Sprague-Dawley rats were obtained from the Animal Experimental Center of Wuhan University (Wuhan, China) and the Center for Disease Control and Prevention of Hubei Province (Wuhan, China). Primary culture of neonatal rat CFs was prepared from ventricles of 1-3 day-old rats via the differential attachment method $(1,2)$. Briefly, following sacrifice, rat hearts were removed from the thorax and immediately placed in $4^{\circ} \mathrm{C} \mathrm{PBS}$, and ventricles were digested with $0.125 \%$ trypsin and $0.08 \%$ collagenase type II at $37^{\circ} \mathrm{C}$ ( 5 times for 5 min each). However, in order to reduce the presence of cell fragments and blood in cell samples, the first digestion solution was discarded. Cells from the last four digestions were collected, and incubated in Dulbecco's modified Eagle's medium/Nutrient Mixture F-12 (DMEM/F12) medium (Hyclone; GE Healthcare, Logan, UT, USA) supplemented with $10 \%$ fetal bovine serum (FBS; Hyclone; GE Healthcare) at $37^{\circ} \mathrm{C}$ for $1 \mathrm{~h}$. Subsequently, unattached or weakly attached cells were discarded, but attached cells were incubated in fresh DMEM/F12 supplemented with $10 \%$ FBS. After the confluence of $\mathrm{CFs}$ reached $80 \%, \mathrm{CF}$ were digested using $0.25 \%$ trypsin and then passaged. Passages 2-4 were used for subsequent experiments in the present study. The cells were cultured in a 6 -well plate when they were used for flow cytometry analysis. The cells were stimulated with TGF- $\beta 1$ or PBS to induce CF transformation into myofibroblasts (1). In the present study, to detect the appropriate concentration of NRG, the effects of several concentrations $(0,10,20,30,40$ and $50 \mu \mathrm{M})$ of NRG on $\alpha$-SMA mRNA levels were evaluated following exposure for $48 \mathrm{~h}$. Next, with the appropriate concentration, the cells were treated with different times $(0$, $12,24,48$ and $72 \mathrm{~h}$ ) to choose the appropriate treatment time. Upon the appropriate concentration and time of NRG, the cells were divided into the following groups: PBS + dimethyl sulfoxide (DMSO), PBS + naringenin, TGF- $\beta 1+$ DMSO and TGF- $\beta 1+$ naringenin. The use of animals in the present study was approved by the Ethics Committee of The First People's Hospital of Yueyang (Yueyang, China).

Immunofluorescence staining. In order to detect the number of CFs that expressed $\alpha$-smooth muscle actin ( $\alpha$-SMA) protein, CFs were stained for the marker $\alpha$-SMA (1). Cells were washed three times with PBS, fixed with $4 \%$ paraformaldehyde at room temperature for $15 \mathrm{~min}$ and permeabilized in $0.2 \%$ Triton X-100 in PBS at room temperature for $20 \mathrm{~min}$. Furthermore, they were blocked with $5 \%$ bovine serum albumin (cat. no. A3733; Sigma-Aldrich; Merck KGaA) in PBS at room temperature for $30 \mathrm{~min}$ and then stained with anti- $\alpha$-SMA antibody (cat. no. ab3280; Abcam, Cambridge, MA, USA; 1:100 dilution) overnight. The cells were incubated with a green fluorescence-marked secondary antibody (cat. no. AS1112; Aspen Biological Co., Ltd., Wuhan, China; 1:100 dilution) for $1 \mathrm{~h}$ at room temperature and then counterstained with DAPI for $8 \mathrm{~min}$ at room temperature. Finally, the cells were covered with mounting medium and kept in the dark at $4^{\circ} \mathrm{C}$.

Cell proliferation assay. Cell proliferation assay was performed according to the manufacturer's instructions of the CCK-8. Initially, the cell suspension was inoculated in a 96-well plate (100 $\mu \mathrm{l} /$ well) and incubated until the confluence of CFs was $60-70 \%$. Following incubation with $30 \mu \mathrm{M}$ NRG at $37^{\circ} \mathrm{C}$ for $48 \mathrm{~h}, \mathrm{CFs}$ were then incubated with CCK- 8 solution (10 $\mu \mathrm{l} /$ well) at $37^{\circ} \mathrm{C}$ for $2 \mathrm{~h}$ and the optical density of each well was measured at $450 \mathrm{~nm}$ using a microplate reader (Tecan Infinite M200; Tecan Group Ltd., Mannedorf, Switzerland). CFs with culture medium and CCK8, without PBS/TGF-h1 or NRG/DMSO treatment, were used as the control.

Flow cytometry analysis. Each group of $\sim 1 \times 10^{6} \mathrm{CFs}$, including PBS + DMSO, PBS + NRG, TGF- $\beta 1+$ DMSO and TGF- $\beta 1+$ NRG, was cultivated in a 6-well plate, collected, fixed in $70 \%$ ice-cold ethanol and, maintained at $4^{\circ} \mathrm{C}$ overnight. CFs were washed with PBS and centrifuged for $2 \mathrm{~min}$ at $716 \mathrm{x} \mathrm{g}$ at room temperature, and the supernatant was discarded. The cell pellet was vortexed in $500 \mu \mathrm{l}$ PBS left behind to avoid clumping of cells. $500 \mu \mathrm{l}$ RNase (cat. no. 10109142001; Sigma-Aldrich; Merck KGaA) was added and the cells were subsequently stained with propidium iodide (PI) (cat. no. P3566; Invitrogen; Thermo Fisher Scientific, Inc.) in the dark for $45 \mathrm{~min}$. To avoid clumps, the cells were transferred through meshed blue-capped falcon tubes prior to analysis using a FACSCalibur system (BD Biosciences, Franklin Lakes, NJ, USA).

Reverse transcription-quantitative polymerase chain reaction assay. CFs were collected and the total mRNA was extracted using TRIzol reagent according to the manufacturer's instructions. cDNA synthesis was performed using the Transcriptor First Strand cDNA synthesis kit (cat. no. 04896866001; 

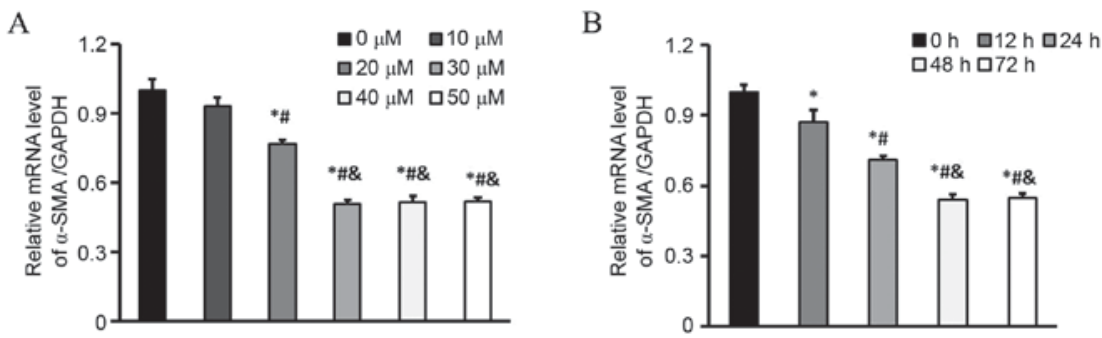

Figure 1. Appropriate concentration and treatment time of NRG in CFs induced by TGF- $\beta 1$. (A) mRNA levels of $\alpha$-SMA in CFs treated with $0,10,2030,40$ and $50 \mu \mathrm{M}$ NRG and stimulated with $5 \mathrm{ng} / \mathrm{ml}$ TGF- $\beta 1$ for $48 \mathrm{~h}$ (n=6 samples per group). ${ }^{*} \mathrm{P}<0.05$ vs. $0 \mu \mathrm{M},{ }^{~}{ }^{*} \mathrm{P}<0.05$ vs. $10 \mu \mathrm{M}$ and ${ }^{~} \mathrm{P}<0.05$ vs. $20 \mu \mathrm{M}$. (B) mRNA levels of $\alpha$-SMA in CFs treated with $30 \mu \mathrm{M} \mathrm{NRG}$ and stimulated with $5 \mathrm{ng} / \mathrm{ml}$ TGF- $\beta 1$ for $0,12,24,48$, and $72 \mathrm{~h}\left(\mathrm{n}=6\right.$ samples per group). ${ }^{*} \mathrm{P}<0.05 \mathrm{vs} .0 \mathrm{~h},{ }^{\prime \prime} \mathrm{P}<0.05$ vs. $12 \mathrm{~h}$ and ${ }^{\&} \mathrm{P}<0.05$ vs. $24 \mathrm{~h}$. NRG, naringenin; CF, cardiac fibroblast; TGF- $\beta 1$, transforming growth factor $\beta 1 ; \alpha$-SMA, $\alpha$-smooth muscle actin.

Roche Diagnostics), according to the manufacturer's protocol. Gene differences were detected using SYBR green (cat. no. 04913850001; Roche Diagnostics) and GAPDH gene expression was used as an internal control. The reaction conditions were as follows: $95^{\circ} \mathrm{C}$ for $10 \mathrm{~min}$, followed by 40 cycles of $95^{\circ} \mathrm{C}$ for $15 \mathrm{sec}$ and $60^{\circ} \mathrm{C}$ for $30 \mathrm{sec}$, then $95^{\circ} \mathrm{C}$ for $15 \mathrm{~min}$ and $60^{\circ} \mathrm{C}$ for $1 \mathrm{~min}$. The expression levels of each gene were quantified through the analysis of the $\mathrm{Cq}$ value and standard curve (14). The sequences of the primer pairs were exhibited as follows: ki67: 5'-TAGAGGATCTGCCTGGCTTC-3' (forward) and 5'-TGTCCTTGGTTGGTTCCTCC-3' (reverse); proliferating cell nuclear antigen (PCNA): 5'-CAACTTGGAATC CCAGAACAGGAG-3' (forward) and 5'-TAAGGTCCCGGC ATATACGTGC-3' (reverse); $\alpha$-SMA: 5'-GCTATTCAGGCT GTGCTGTC-3' (forward) and 5'-GGTAGTCGGTGAGAT CTCGG-3' (reverse); connective tissue growth factor (CTGF): 5'-GGAAGACACATTTGGCCCTG-3' (forward) and 5'-GCA ATTTTAGGCGTCCGGAT-3' (reverse); collagen I: 5'-GAG CGGAGAGTACTGGATCGA-3' (forward) and 5'-CTGACC TGTCTCCATGTTGCA-3' (reverse); collagen III: 5'-TGC CATTGCTGGAGTTGGA-3' (forward) and 5'-GAAGACATG ATCTCCTCAGTGTTGA-3' (reverse) and GAPDH: 5'-GGG TGATGCTGGTGCTGAGTATGT-3' (forward) and 5'-CAG TGGATGCAGGGATGATGTTCT-3' (reverse).

Western blot analysis. Proteins were extracted from CFs using radioimmunoprecipitation assay lysis buffer (cat. no. P0013B; Beyotime Institute of Biotechnology, Haimen, China), and the protein concentration was determined using a BCA protein assay kit (cat. no. 23227; Pierce; Thermo Fisher Scientific, Inc.). Proteins were loaded (10 $\mu \mathrm{g} /$ well) and separated using $10 \%$ SDS-PAGE at $120 \mathrm{~V}$ for $120 \mathrm{~min}$. Subsequently, the proteins were transferred onto polyvinyl difluoride transfer membranes at $4^{\circ} \mathrm{C}$ and the membranes were blocked for $60 \mathrm{~min}$ with freshly prepared 5\% skimmed milk in Tris-buffered saline with Tween 20 (TBST) at room temperature. Following this, the membranes were incubated with various primary antibodies, including cyclin D1 (1:1,000), CDK4 (1:1,000), CDK6 $(1: 1,000)$, cyclin E2 $(1: 500)$, CDK2 $(1: 1,000)$ and GAPDH $(1: 10,000)$ overnight at $4^{\circ} \mathrm{C}$ with gentle shaking. Membranes were washed three times with TBST, and incubated with either HRP-conjugated goat anti-mouse (cat. no. 074-1806; Kirkegaard and Perry Laboratories, Inc., Gaithersburg, MD, USA; 1:10,000 dilution) or goat anti-rabbit (cat. no. 074-1506; Kirkegaard and Perry Laboratories, Inc.; 1:10,000 dilution) secondary antibodies for $60 \mathrm{~min}$ at room temperature with shaking. Following four washes of the membranes, images were captured on films, which were placed in LumiGLO solution (cat. no. 7003; Cell Signaling Technology, Inc.) for $1 \mathrm{~min}$ at room temperature. Following development, the images were placed into an automatic image analyzer to determine the expression levels of the proteins as well as the reference gray scale values. GAPDH was used as a loading control and three independent experiments were performed.

Statistical analysis. Data are expressed as the mean \pm standard error of the mean. Statistical analyses were performed using SPSS version 13.0 software (SPSS, Inc., Chicago, IL, USA). Comparisons between each group were performed using one-way analysis of variance followed by Fisher's least significant difference test. $\mathrm{P}<0.05$ was considered to indicate a statistically significant difference.

\section{Results}

Appropriate concentration and treatment time of NRG in CFs induced by $T G F-\beta 1$. Initially, to detect the appropriate concentration of NRG, the effects of several concentrations of it were measured on the $\alpha$-SMA mRNA level following exposure for $48 \mathrm{~h}$. It was revealed that induction of $\alpha$-SMA expression was significantly inhibited when NRG was added at a concentration of $30 \mu \mathrm{M}$, compared with that when 0,10 or $20 \mu \mathrm{M} \mathrm{NRG}$ was added (Fig. 1A). Additionally, it was demonstrated that $30 \mu \mathrm{M}$ NRG inhibited $\alpha$-SMA expression in a time-dependent manner. Compared with the 12 and 24 h groups, the minimum expression of $\alpha$-SMA was revealed at the 48 and $72 \mathrm{~h}$ time points (Fig. 1B). Therefore, $30 \mu \mathrm{M}$ NRG with $48 \mathrm{~h}$ treatment time was selected for subsequent experiments.

NRG treatment inhibits transformation and proliferation of CFs. Transformation of CFs into cardiac myofibroblasts was investigated with $\alpha$-SMA. The expression of $\alpha$-SMA protein detected via immunofluorescence staining was markedly inhibited in the NRG-treated group, which indicated that NRG treatment for $48 \mathrm{~h}$ was able to reduce the number of CFs that expressed $\alpha$-SMA protein induced by TGF- $\beta 1$ (Fig. $2 \mathrm{~A}$ ). $\mathrm{CF}$ proliferation was investigated via CCK-8 assay (Fig. 2B). Subsequently, the effect of NRG on proliferation of CFs was further assessed by the mRNA levels of ki67 and PCNA (Fig. 2C and D). It was revealed that the mRNA levels of ki67 
A

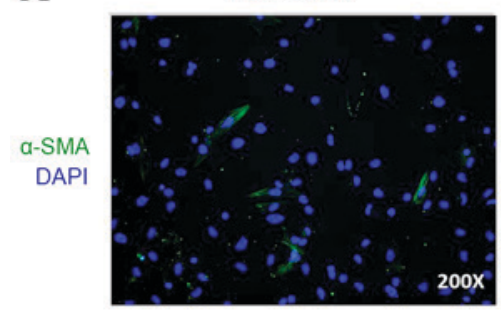

B

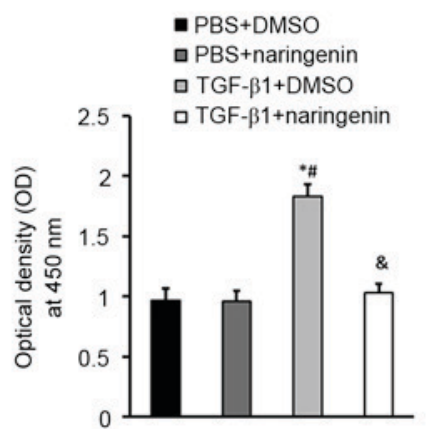

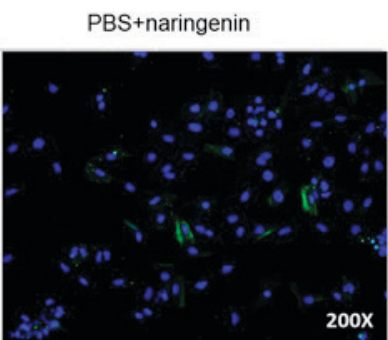

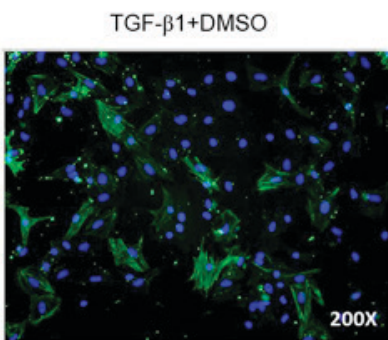

TGF- $\beta 1+$ naringenin

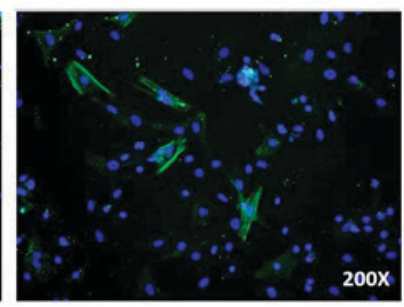

$\mathrm{C}$

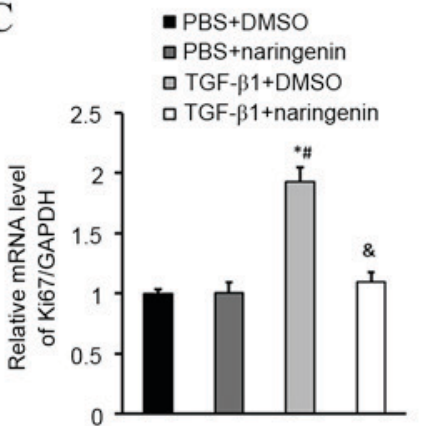

$\mathrm{D}$

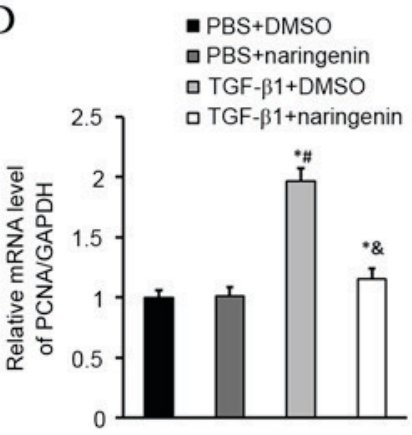

Figure 2. NRG treatment inhibits transformation and proliferation of CFs. (A) Representative immunofluorescent stained images of $\alpha$-SMA in CFs treated with or without NRG ( $\mathrm{n}=3$ independent experiments). (B) Cell counting kit- 8 assay results revealed that the proliferation of CFs stimulated with TGF- $\beta 1$ was inhibited by NRG ( $\mathrm{n}=6$ samples per group). (C and D) mRNA levels of ki67 and PCNA in CFs of the indicated groups ( $\mathrm{n}=6$ samples per group). * $<0.05$ vs. PBS + DMSO, ${ }^{\text {}} \mathrm{P}<0.05$ vs. PBS + NRG and ${ }^{\&} \mathrm{P}<0.05$ vs. TGF- $\beta 1+$ DMSO. NRG, naringenin; $\mathrm{CF}$, cardiac fibroblast; $\alpha-$ SMA, $\alpha$-smooth muscle actin; TGF- $\beta 1$, transforming growth factor $\beta 1$; DMSO, dimethyl sulfoxide; PCNA, proliferating cell nuclear antigen.

A

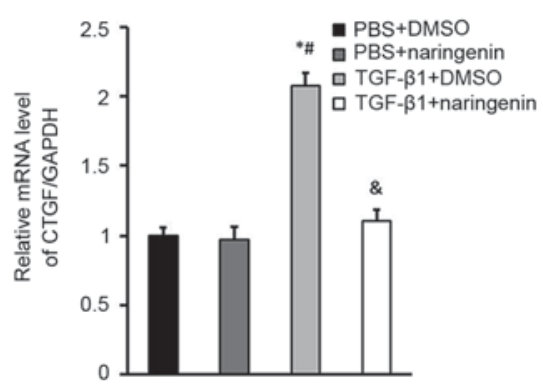

B

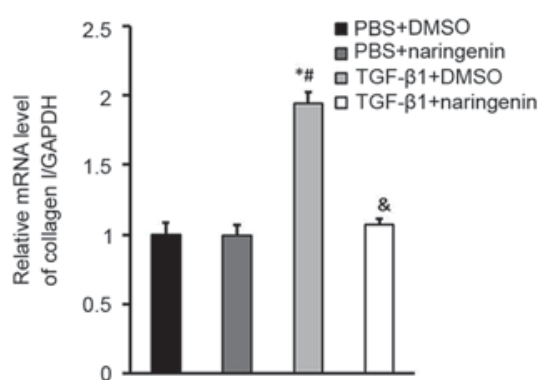

$\mathrm{C}$

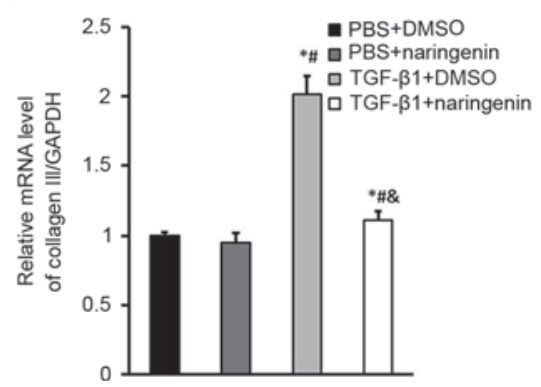

Figure 3. NRG treatment inhibits collagen synthesis of CF. Relative mRNA levels of (A) CTGF, (B) collagen I and (C) collagen III in CFs of the indicated groups ( $\mathrm{n}=6$ samples per group). ${ }^{*} \mathrm{P}<0.05$ vs. $\mathrm{PBS}+\mathrm{DMSO},{ }^{*} \mathrm{P}<0.05$ vs. $\mathrm{PBS}+\mathrm{NRG}$ and ${ }^{\&} \mathrm{P}<0.05$ vs. TGF- $\beta 1+\mathrm{DMSO}$. NRG, naringenin; $\mathrm{CF}$, cardiac fibroblast; CTGF, connective tissue growth factor; TGF- $\beta 1$, transforming growth factor $\beta 1$; DMSO, dimethyl sulfoxide.

and PCNA were significantly inhibited in CFs following treatment with NRG compared with the TGF- $\beta 1$ group $(\mathrm{P}<0.05)$, which indicated that NRG treatment was able to inhibit the proliferation of CFs compared with the TGF- $\beta 1$ group.

$N R G$ treatment inhibits collagen synthesis of CFs. Collagen synthesis of cardiac myofibroblasts is perceived to be important in the development of cardiac fibrosis $(15,16)$. Therefore, the role of NRG on collagen synthesis was detected via the mRNA levels of CTGF, collagen I and collagen III. It was revealed that NRG significantly decreased the mRNA levels of CTGF, collagen I and collagen III in CFs induced by TGF- $\beta 1$ (Fig. 3).

$N R G$ treatment induces CF cell cycle arrest in the G0/G1-phase. In order to elucidate the mechanism of $\mathrm{CF}$ proliferation regulated by NRG, the effect of NRG on cell cycle progression was evaluated. CFs under subconfluent culture conditions were synchronized by serum starvation, which caused cell cycle arrest at the G0/G1-phase, and then treated with the indicated stimuli, labeled with PI and analyzed by flow cytometry $(17,18)$. It was revealed that the $\mathrm{S}$-phase population was significantly increased and the G0/G1-phase was significantly decreased in the TGF- $\beta 1+$ DMSO group compared with the PBS groups, whereas the G0/G1-phase of CFs in the TGF- $\beta 1$ group treated with NRG was significantly increased and the S-phase population was significantly lower compared with all other groups. These results indicated that NRG treatment induces CF cell cycle arrest in the G0/G1-phase (Table I).

$N R G$ inhibits CFs entering the S-phase by downregulating the cyclin D1-CDK4/6 and cyclin E2-CDK2 complexes. The G0/G1 to S-phase transition is primarily regulated by the cyclin D1-CDK4/6 and cyclin E2-CDK2 complexes $(19,20)$. To investigate the status of these complexes in NRG-treated CFs induced by TGF- $\beta 1$, the protein levels of individual cyclins 
Table I. NRG treatment induces cardiac fibroblast cell cycle arrest in the G0/G1-phase (\%).

\begin{tabular}{lrrrr}
\hline Stage & PBS + DMSO & PBS + NRG & TGF- $\beta 1+$ DMSO & TGF- $\beta 1+$ NRG \\
\hline G0/G1 & $77.32 \pm 3.11$ & $76.08 \pm 3.32$ & $65.641 \pm 2.90^{\mathrm{a}, \mathrm{b}}$ & $86.53 \pm 2.64^{\mathrm{a}-\mathrm{c}}$ \\
S & $15.61 \pm 1.18$ & $16.31 \pm 0.93$ & $25.87 \pm 1.47^{\mathrm{a}, \mathrm{b}}$ & $10.19 \pm 0.78^{\mathrm{a}-\mathrm{c}}$ \\
G2/M & $7.07 \pm 0.34$ & $7.61 \pm 0.73$ & $8.74 \pm 0.69^{\mathrm{a}}$ & $3.27 \pm 0.48^{\mathrm{a}-\mathrm{c}}$
\end{tabular}

DNA flow cytometry analysis revealed an increased population of the G0/G1-phase and smaller S-phase in the TGF- $\beta 1+$ NRG compared with the TGF- $\beta 1+$ DMSO group. ${ }^{\mathrm{a}} \mathrm{P}<0.05$ vs. PBS + DMSO, ${ }^{\mathrm{b}} \mathrm{P}<0.05$ vs. $\mathrm{PBS}+\mathrm{NRG}$ and ${ }^{\mathrm{c}} \mathrm{P}<0.05$ vs. TGF- $\beta 1+\mathrm{DMSO}$. NRG, naringenin; DMSO, dimethyl sulfoxide; TGF- $\beta 1$, transforming growth factor $\beta 1$.
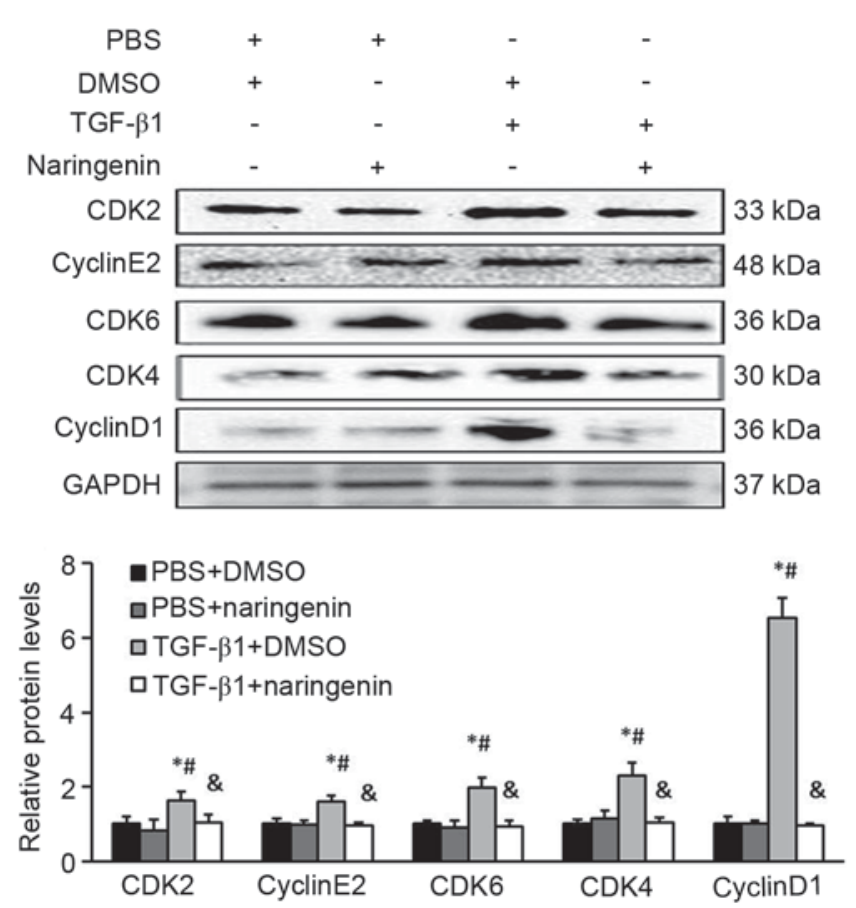

Figure 4. NRG inhibits CFs from entering the S-phase via downregulating the cyclin D1-CDK4/6 and cyclin E2-CDK2 complexes. Representative western blot analysis and statistical results of the protein levels of cyclin D1, $\mathrm{CDK} 4, \mathrm{CDK} 6$, cyclin $\mathrm{E} 2$ and CDK2 in CFs of the indicated groups $(\mathrm{n}=3$ independent experiments). ${ }^{*} \mathrm{P}<0.05$ vs. $\mathrm{PBS}+\mathrm{DMSO},{ }^{\#} \mathrm{P}<0.05$ vs. $\mathrm{PBS}+\mathrm{NRG}$ and ${ }^{\&} \mathrm{P}<0.05$ vs. TGF- $\beta 1+$ DMSO. NRG, naringenin; $\mathrm{CF}$, cardiac fibroblast; DMSO, dimethyl sulfoxide; TGF- $\beta 1$, transforming growth factor $\beta 1$; CDK, cyclin dependent kinase.

and CDKs were measured by western blot analysis. It was revealed that the expression levels of cyclin D1, CDK4, CDK6, cyclin E2 and CDK2 decreased significantly in CFs treated with NRG and TGF- $\beta 1$ compared with the TGF- $\beta 1$ group, which indicated that NRG repressed the G1/S-phase transition, in part, by downregulating the cyclin D1-CDK4/6 and cyclin E2-CDK2 complexes (Fig. 4).

\section{Discussion}

In the present study, the effects and the probable mechanism of NRG on CFs stimulated with TGF- $\beta 1$ in vitro were investigated. It was revealed that NRG had the following effects: i) Inhibited $\mathrm{CF}$ transformation into cardiac myofibroblasts; ii) inhibited the proliferation of CFs; iii) inhibited the synthesis of CTGF, collagen I and III in CFs stimulated with TGF- $\beta 1$; iv) induced
CF cell cycle arrest in the G0/G1-phase; and v) repressed the G1/S-phase transition of CFs partly via downregulating the cyclin D1-CDK4/6 and cyclin E2-CDK2 complexes following TGF- $\beta 1$ induction. Although further studies are required, to the best of our knowledge the present study is the first to link the anti-proliferative effect of NRG on CFs with the inhibition of DNA synthesis via G0/G1 arrest, indicating that NRG may be used as a therapeutic agent to regulate cardiac fibrosis in the future.

Cardiac fibrosis is associated with various physiological causes, including CF proliferation, inflammation and hypertension (4). Among these causes, the transformation of CFs into cardiac myofibroblasts and activated proliferation are known to be important events in the development of cardiac fibrosis, which may promote pathological hypertrophy and fibrosis and subsequently result in cardiac remodeling and cardiac dysfunction (6). Therefore, inhibiting CF proliferation is considered important for treating cardiovascular diseases. In the present study, the anti-proliferative effect of NRG was examined on CF proliferation. NRG significantly inhibited TGF- $\beta 1$-induced CF proliferation, transformation into cardiac myofibroblasts and the synthesis of CTGF, collagen I and III of CFs. Notably, the anti-proliferative effect of NRG was associated with the inhibition of DNA synthesis via G0/G1 cell cycle arrest. The cell cycle consists of four sequential phases including $\mathrm{G} 0 / \mathrm{G} 1$, S, G2 and M. It regulates cellular proliferation by a highly controlled process involving a complex cascade of cellular events, including regulatory factor cyclins and CDKs $(21,22)$. The cyclin D1-CDK4/6 and cyclin E2-CDK2 complexes are important mediators of the cell cycle transition from the G0/G1 to S-phase $(19,20)$. In the present study, NRG not only inhibited cyclin D1 and cyclin E2 expression, it also inhibited CDK2/4 and CDK6 expression. Similar to the present study, Lee et al (21) demonstrated previously that (2S)-NRG inhibited the platelet-derived growth factor (PDGF)-BB-induced proliferation of vascular smooth muscle cells via a G0/G1 arrest, did not affect the signaling pathways associated with PDGF-R $\beta$, protein kinase $B$, extracellular signal-regulated kinase $1 / 2$ or phospholipase C- $\gamma 1$ but downregulated the expression of cyclin D and $\mathrm{E}, \mathrm{CDK} 2, \mathrm{CDK} 4$ and reduced retinoblastomaprotein phosphorylation. This indicated that (2S)-NRG may be valuable as a therapeutic agent for managing atherosclerosis and/or vascular restenosis.

In conclusion, the results of the present study demonstrate that NRG was able to inhibit TGF- $\beta 1$-induced proliferation of CFs via G0/G1 arrest, resulting in the downregulated expression 
of cyclin D1-CDK4/6 and cyclin E2-CDK2. Therefore, the results indicated that NRG may be used as a novel treatment strategy due to its anti-fibrotic effect in CFs by regulating proliferation, transformation and collagen synthesis of CFs in response to pathological stress. However, the detailed mechanism of how NRG regulates cardiac remodeling remains to be demonstrated in animal models. Additionally, detailed regulatory mechanisms of cyclins and CDKs or other signaling pathways, which are associated with the process of NRG affecting cardiac fibrosis, require further study.

\section{Acknowledgements}

The authors would like to thank all members of the Department of Cardiology at The First People Hospital of Yueyang (Yueyang, China) for their expert technical assistance and advice.

\section{References}

1. Yi X, Li X, Zhou Y, Ren S, Wan W, Feng G and Jiang X: Hepatocyte growth factor regulates the TGF- $\beta 1$-induced proliferation, differentiation and secretory function of cardiac fibroblasts. Int J Mol Med 34: 381-390, 2014.

2. Zhou Y, Yi X, Wang T and Li M: Effects of angiotensin II on transient receptor potential melastatin 7 channel function in cardiac fibroblasts. Exp Ther Med 9: 2008-2012, 2015.

3. Li M, Yi X, Ma L and Zhou Y: Hepatocyte growth factor and basic fibroblast growth factor regulate atrial fibrosis in patients with atrial fibrillation and rheumatic heart disease via the mitogen-activated protein kinase signaling pathway. Exp Ther Med 6: 1121-1126, 2013

4. Zhou YM, Li MJ, Zhou YL, Ma LL and Yi X: Growth differentiation factor-15 (GDF-15), novel biomarker for assessing atrial fibrosis in patients with atrial fibrillation and rheumatic heart disease. Int J Clin Exp Med 8: 21201-21207, 2015.

5. Stempien-Otero A, Kim DH and Davis J: Molecular networks underlying myofibroblast fate and fibrosis. J Mol Cell Cardiol 97: $153-161,2016$.

6. Travers JG, Kamal FA, Robbins J, Yutzey KE and Blaxall BC: Cardiac fibrosis: The fibroblast awakens. Circ Res 118: 1021-1040, 2016.

7. Moore-Morris T, Cattaneo P, Puceat M and Evans SM: Origins of cardiac fibroblasts. J Mol Cell Cardiol 91: 1-5, 2016.

8. van Putten S, Shafieyan Y and Hinz B: Mechanical control of cardiac myofibroblasts. J Mol Cell Cardiol 93: 133-142, 2016.
9. Pinho-Ribeiro FA, Zarpelon AC, Mizokami SS, Borghi SM, Bordignon J, Silva RL, Cunha TM, Alves-Filho JC, Cunha FQ, Casagrande R and Verri WA Jr: The citrus flavonone naringenin reduces lipopolysaccharide-induced inflammatory pain and leukocyte recruitment by inhibiting NF- $\mathrm{B}$ activation. J Nutr Biochem 33: 8-14, 2016.

10. Xing BH, Yang FZ and $\mathrm{Wu} \mathrm{XH}$ : Naringenin enhances the efficacy of human embryonic stem cell-derived pancreatic endoderm in treating gestational diabetes mellitus mice. J Pharmacol Sci 131: 93-100, 2016.

11. Zhang F, Dong W, Zeng W, Zhang L, Zhang C, Qiu Y, Wang L, Yin X, Zhang C and Liang W: Naringenin prevents TGF- $\beta 1$ secretion from breast cancer and suppresses pulmonary metastasis by inhibiting PKC activation. Breast Cancer Res 18: 38, 2016.

12. Zhang N, Yang Z, Yuan Y, Li F, Liu Y, Ma Z, Liao H, Bian Z, Zhang Y, Zhou H, et al: Naringenin attenuates pressure overload-induced cardiac hypertrophy. Exp Ther Med 10: 2206-2212, 2015.

13. Chtourou Y, Slima AB, Makni M, Gdoura R and Fetoui H: Naringenin protects cardiac hypercholesterolemia-induced oxidative stress and subsequent necroptosis in rats. Pharmacol Rep 67: 1090-1097, 2015.

14. Livak KJ and Schmittgen TD: Analysis of relative gene expression data using real-time quantitative PCR and the 2(-Delta Delta C(T)) method. Methods 25: 402-408, 2001.

15. Roche P and Czubryt MP: Transcriptional control of collagen I gene expression. Cardiovasc Hematol Disord Drug Targets 14: 107-120, 2014.

16. Li AH, Liu PP, Villarreal FJ and Garcia RA: Dynamic changes in myocardial matrix and relevance to disease: Translational perspectives. Circ Res 114: 916-927, 2014.

17. Langan TJ and Chou RC: Synchronization of mammalian cell cultures by serum deprivation. Methods Mol Biol 761: 75-83, 2011.

18. Lin X, Yang X, Li Q, Ma Y, Cui S, He D, Lin X, Schwartz RJ and Chang J: Protein tyrosine phosphatase-like A regulates myoblast proliferation and differentiation through MyoG and the cell cycling signaling pathway. Mol Cell Biol 32: 297-308, 2012.

19. Lundberg AS and Weinberg RA: Functional inactivation of the retinoblastoma protein requires sequential modification by at least two distinct cyclin-cdk complexes. Mol Cell Biol 18: 753-761, 1998.

20. Lauper N, Beck AR, Cariou S, Richman L, Hofmann K, Reith W, Slingerland JM and Amati B: Cyclin E2: A novel CDK2 partner in the late $\mathrm{G} 1$ and $\mathrm{S}$ phases of the mammalian cell cycle. Oncogene 17: 2637-2643, 1998.

21. Lee JJ, Yi H, Kim IS, Nhiem NX, Kim YH and Myung CS: (2S)-naringenin from Typha angustata inhibits vascular smooth muscle cell proliferation via a G0/G1 arrest. J Ethnopharmacol 139: 873-878, 2012.

22. Bendris N, Lemmers B and Blanchard JM: Cell cycle, cytoskeleton dynamics and beyond: The many functions of cyclins and CDK inhibitors. Cell Cycle 14: 1786-1798, 2015. 